\title{
Long-term use of implanted peroneal functional electrical stimulation for stroke- affected gait: the effects on muscle and motor nerve
}

\author{
Frank Berenpas ${ }^{1}$, Vivian Weerdesteyn ${ }^{1}$, Alexander C. Geurts ${ }^{1 *+}$ (I) and Nens van Alfen ${ }^{2+}$
}

\begin{abstract}
Background: Peripheral changes to muscle and motor nerves occur following stroke, which may further impair functional capacity. We investigated whether a year-long use of an implanted peroneal FES system reverses strokerelated changes in muscles and motor nerves in people with foot drop in the chronic phase after supratentorial stroke.

Methods: Thirteen persons with a chronic stroke (mean age 56.1 years, median Fugl-Meyer Assessment leg score 71\%) were included and received an implanted peroneal FES system (ActiGait ${ }^{\oplus}$ ). Quantitative muscle ultrasound (QMUS) images were obtained bilaterally from three leg muscles (i.e. tibialis anterior, rectus femoris, gastrocnemius). Echogenicity (muscle ultrasound gray value) and muscle thickness were assessed over a one-year follow-up and compared to age-, sex-, height- and weight-corrected reference values. Compound motor action potentials (CMAPs) and motor evoked potentials (MEPs) were obtained from the tibialis anterior muscle. Generalized estimated equation modeling was used to assess changes in QMUS, CMAPs and MEPs outcomes over the follow-up period.
\end{abstract}

Results: Echogenicity of the tibialis anterior decreased significantly during the follow-up on the paretic side. Z-scores changed from 0.88 at baseline to -0.15 after 52 weeks. This was accompanied by a significant increase in muscle thickness on the paretic side, where $z$-scores changed from -0.32 at baseline to 0.48 after 52 weeks. Echogenicity of the rectus femoris normalized on both the paretic and non-paretic side (z-scores changed from -1.09 and -1.51 to 0.14 and -0.49 , respectively). Amplitudes of CMAP and MEP (normalized to CMAP) were reduced during follow-up, particularly on the paretic side ( $\triangle C M A P=20 \%$ and $\triangle M E P=14 \%)$.

Conclusions: We show that the structural changes to muscles following stroke are reversible with FES and that these changes might not be limited to electrically stimulated muscles. No evidence for improvement of the motor nerves was found.

Keywords: Stroke, Foot drop, Implanted FES, Ultrasound, TMS, Transsynaptic degeneration

\section{Introduction}

Stroke is typically defined as a lesion of the upper motor neuron (UMN). However, it is known that secondary to this UMN lesion peripheral changes occur after a stroke $[1,2]$. Following the loss of central activation, lower motor neurons (LMN) may become functionally depressed or

\footnotetext{
* Correspondence: Sander.Geurts@radboudumcnl.

${ }^{+}$Alexander C. Geurts and Nens van Alfen contributed equally to this work.

'Department of Rehabilitation, Donders Institute for Brain, Cognition and

Behaviour, Radboud University Medical Center, P.O. Box 9101, 6500, HB,

Nijmegen, The Netherlands

Full list of author information is available at the end of the article
}

may even undergo 'transsynaptic degeneration' leading to denervation of muscle fibers [3-5]. Since denervation of muscle fibers induces muscle atrophy and infiltration of fibrous tissue and fat, this process of denervation after stroke also has an effect on the structure of skeletal muscles. Indeed, muscle atrophy and infiltration of fibrous tissue and fat are often reported in paretic muscles after stroke [6-8]. Recently we have shown that structural changes to muscles after stroke are not restricted to the paretic side alone and that the changes in muscle structure cannot be explained solely by disuse [9]. These

(c) The Author(s). 2019 Open Access This article is distributed under the terms of the Creative Commons Attribution 4.0 International License (http://creativecommons.org/licenses/by/4.0/), which permits unrestricted use, distribution, and reproduction in any medium, provided you give appropriate credit to the original author(s) and the source, provide a link to the Creative Commons license, and indicate if changes were made. The Creative Commons Public Domain Dedication waiver (http://creativecommons.org/publicdomain/zero/1.0/) applies to the data made available in this article, unless otherwise stated. 
changes to skeletal muscle structure are believed to further impair functional capacity of people with stroke [8]. Therefore, interventions preventing or mitigating this undesirable involvement of the peripheral nerve system and muscles after stroke are needed.

Functional electrical stimulation (FES) of paretic muscles is a commonly applied method to compensate for severe muscle weakness after stroke. One of the most widely used applications of FES in people with stroke is the activation of the peroneal nerve to reduce foot drop [10]. With peroneal FES, paretic dorsiflexor muscles are electrically activated during the swing phase and early stance of the gait cycle, resulting in an 'active' foot elevation [11]. It has been theorized that such electrically induced contractions combined with voluntary contractions can strengthen spinal synapses and induce cortical changes [12]. Indeed, increased excitability, metabolism and reorganization of the motor cortex have been reported after prolonged peroneal FES use in people with a neurological disease, including people with stroke [1316]. These plastic changes after long-term FES use indicate central motor recovery, which raises the question whether stroke-related changes to skeletal muscles and lower motor neurons can also be reversed with prolonged FES use.

In this study we aimed to investigate whether a yearlong use of an implanted peroneal FES system (ActiGait', Neurodan, Denmark, Otto Bock Group, 2006) reverses stroke-related changes in skeletal muscles and their motor innervation in people with persistent foot drop in the chronic phase after a supratentorial stroke. Bilateral leg muscle thickness and echogenicity were monitored over time using quantitative muscle ultrasound (QMUS), which is valid method for objective assessment of muscle architecture [17-19] and has been used in various patient populations with neuromuscular and central nervous system disorders $[9,20$, 21]. Muscle thickness provides an indication of the presence of muscle atrophy (or hypertrophy), whereas echogenicity (i.e. how white or black the image looks on the screen) is a measure of how (ab-) normal the tissue architecture is. Infiltration of muscle fibers with fat and fibrous tissue following denervation increases muscle echogenicity, making the muscle appear more white on the screen (see Fig. 1 for an example). Furthermore, whether long-term FES stimulation of ankle dorsiflexor muscles changes functioning of the lower motor neurons and alters corticospinal integrity was tested by obtaining compound motor action potentials (CMAPs) and motor evoked potentials (MEPs) from the primary ankle dorsiflexor muscle (i.e. tibialis anterior muscle).

We hypothesized that, following a year-long period of implanted peroneal FES use, muscle architecture would be restored in the electrically stimulated ankle dorsiflexor muscles, but not in other muscles on the paretic or non-paretic side. In addition, as long-term use of FES has been found to induce cortical changes, we hypothesized that MEPs would be increased after a year of FES use. The results of this study will lead to a better insight into the reversibility of architectural changes of muscles and motor nerves after stroke using long-term FES.

\section{Methods \\ Participants}

All participants were recruited from the outpatient clinic of the department of Rehabilitation at the Radboud university medical center in Nijmegen and were enrolled in a study evaluating the effects of implanted functional electrical stimulation (FES) on ambulation [22]. Inclusion criteria were: (1) having sustained a supratentorial stroke, i.e. a lesion in one of the cerebral hemispheres, at least 6 months before inclusion, (2) paresis including unilateral foot dorsiflexion weakness (Medical Research Council scale <5), (3) the ability to walk at least $10 \mathrm{~m}$ without a walking aid (except for the use of an anklefoot orthosis which was allowed) and [4] a positive response to surface-based peroneal nerve stimulation (NESS L300, Bioness inc, Valencia, California) defined as the ability to make initial heel contact during gait with stimulation. Subjects were excluded if: (1) they had a history of (poly-) neuropathy or (poly-) radiculopathy, or (2) were morbidly obese (body mass index $>40$ ), as this can preclude reliable QMUS measurements by attenuation of the ultrasound beam, or (3) reported contraindications for transcranial magnetic stimulation (TMS) (e.g. active epilepsy or the presence of an implanted pacemaker, neurostimulator or cochlear implant). All patients gave their informed consent. The study was approved by the local medical ethics committee and conducted in accordance with the World Medical Association Declaration of Helsinki [23].

\section{Demographic and clinical characteristics}

Age, sex, time since stroke, type of stroke, body mass index, leg motor strength (Motricity Index [24]) and leg motor selectivity (Fugl-Meyer Assessment [25]) were obtained by the same rehabilitation physician (ACG) at inclusion. In addition, preferred walking speed [26] at baseline and after 52 weeks of implanted FES use was assessed on a 10-m walkway using a stopwatch. Physical activity at baseline and after 52 weeks of FES use was registered by means of a Digi-Walker SW-650 pedometer (Yamax Corporation, Tokyo, Japan) counting the amount of daily steps averaged over a period of 7 days. 


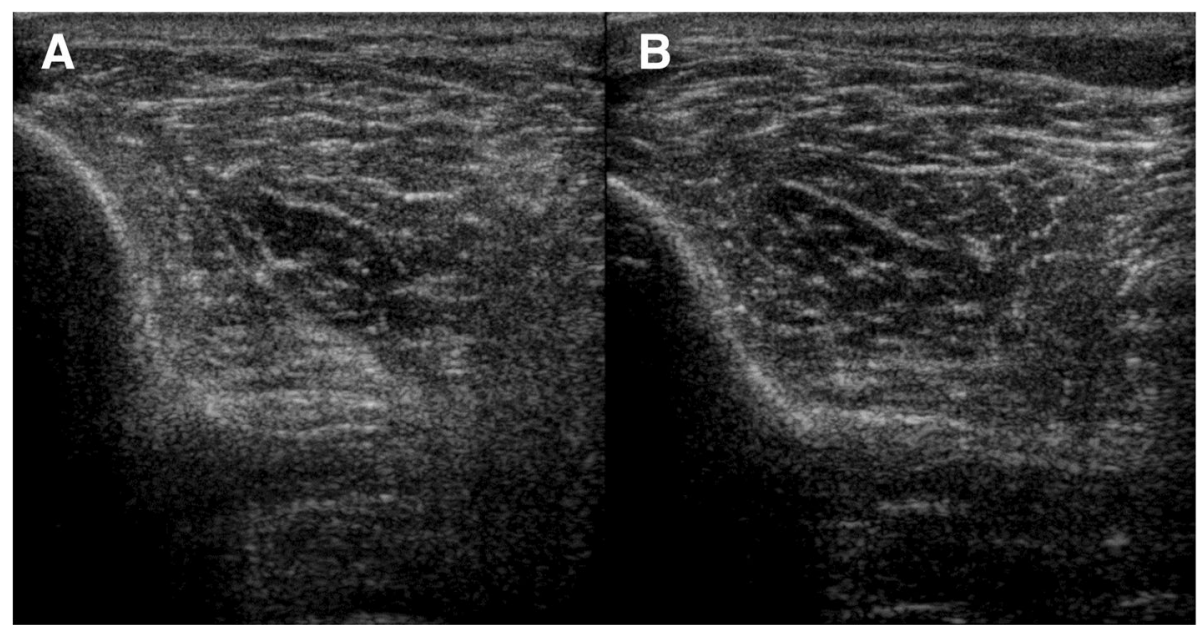

Fig. 1 Example of paretic side $(\mathbf{a})$ and non-paretic side $(\mathbf{b})$ tibialis anterior. The paretic tibialis anterior muscle appears whiter on the screen (i.e. a higher echogenicity), indicating infiltration of intramuscular fat and fibrous tissue. Echogenicity $z$-scores were $z=1.45$ and $z=0.29$ for the paretic and non-paretic side, respectively

\section{ActiGait ${ }^{\circledR}$ implanted FES system}

The ActiGait $^{\circ}$ system is an implantable 4-channel peroneal nerve stimulator (Fig. 2). The implant consists of an electrode cuff at the distal end and a stimulator body at the proximal end connected by a lead wire. The electrode cuff has 4 separate electrodes, which are selectively controlled by the stimulator body, allowing differential activation of nerve fibers to the tibialis anterior, peroneus longus/brevis, and toe extensor muscles. The system is operated through external parts: a heel switch (placed under the heel and attached to the shoe or a special sock) and a control unit, worn at the pelvis, which is connected to an antenna on the skin directly over the stimulator body. The control unit enables users to switch the stimulation on and off and to make adjustments in stimulation intensity. The heel switch wirelessly communicates with the control unit to provide information for onset and offset of stimulation.

ActiGait implantation was performed by a trained neurosurgeon at the Radboud university medical center in Nijmegen. The implant was activated 3 weeks after surgery. Use of the Acitgait ${ }^{\circ}$ system was then built up gradually in 3 weeks from 15 to $60 \mathrm{~min}$ per day in the first week up to minimally $6 \mathrm{~h}$ per day in the third week. The procedure of ActiGait ${ }^{\circ}$ implantation and system activation has previously been described in detail [27-29] and can be found in the Additional file 1 of this report.

\section{Study design}

A within-subjects repeated-measures design was used for the follow-up of 1 year after implantation. QMUS measurements were performed at inclusion (T0) as well as 26 weeks (T3) and 52 weeks (T4) after activation of the ActiGait $^{\circ}$ system. Neurophysiologic assessments were performed at inclusion (T0) and 2 weeks (T1), 8 weeks (T2), 26 weeks (T3) and 52 weeks (T4) after activation of the ActiGait system. The effects of long-term implanted FES use on walking capacity have been published elsewhere [22, 29].

\section{Measurements \\ Quantitative muscle ultrasound}

We used QMUS, a method with high interrater reliability [30-32], to assess the primary ankle dorsiflexor muscle (i.e., the tibialis anterior muscle), its antagonist (i.e., the medial head of the gastrocnemius muscle) and the rectus femoris muscle, a biarticular upper leg muscle, all being key muscles for locomotion. Muscle ultrasound images of the tibialis anterior, medial head of the gastrocnemius, and rectus femoris muscles were obtained bilaterally with a Philips IU 22 ultrasound machine (Philips Healthcare Systems, Best, The Netherlands) and a broadband linear 5-17 MHz transducer. A dedicated musculoskeletal preset was used for all scanning, with fixed system parameters set to: gain $70 \mathrm{~dB}$, compression 55 , time gain in neutral position, and focal range of 1.0 to $2.5 \mathrm{~cm}$ depth. To ensure reproducibility, automatic image optimizing software was turned off as much as possible. System settings remained unchanged throughout all measurements [33]. Muscle ultrasound images were taken at predefined sites corresponding to the maximum muscle thickness of each muscle, following the description of Scholten et al. [34]. At each assessment, three separate muscle ultrasound images were taken from each muscle, all the while ensuring there was no pressure on the skin or the muscle and with the participant in a relaxed, supine position. The digital images were stored as DICOM files for offline analysis. In each image a region of interest (ROI) was selected. Using 


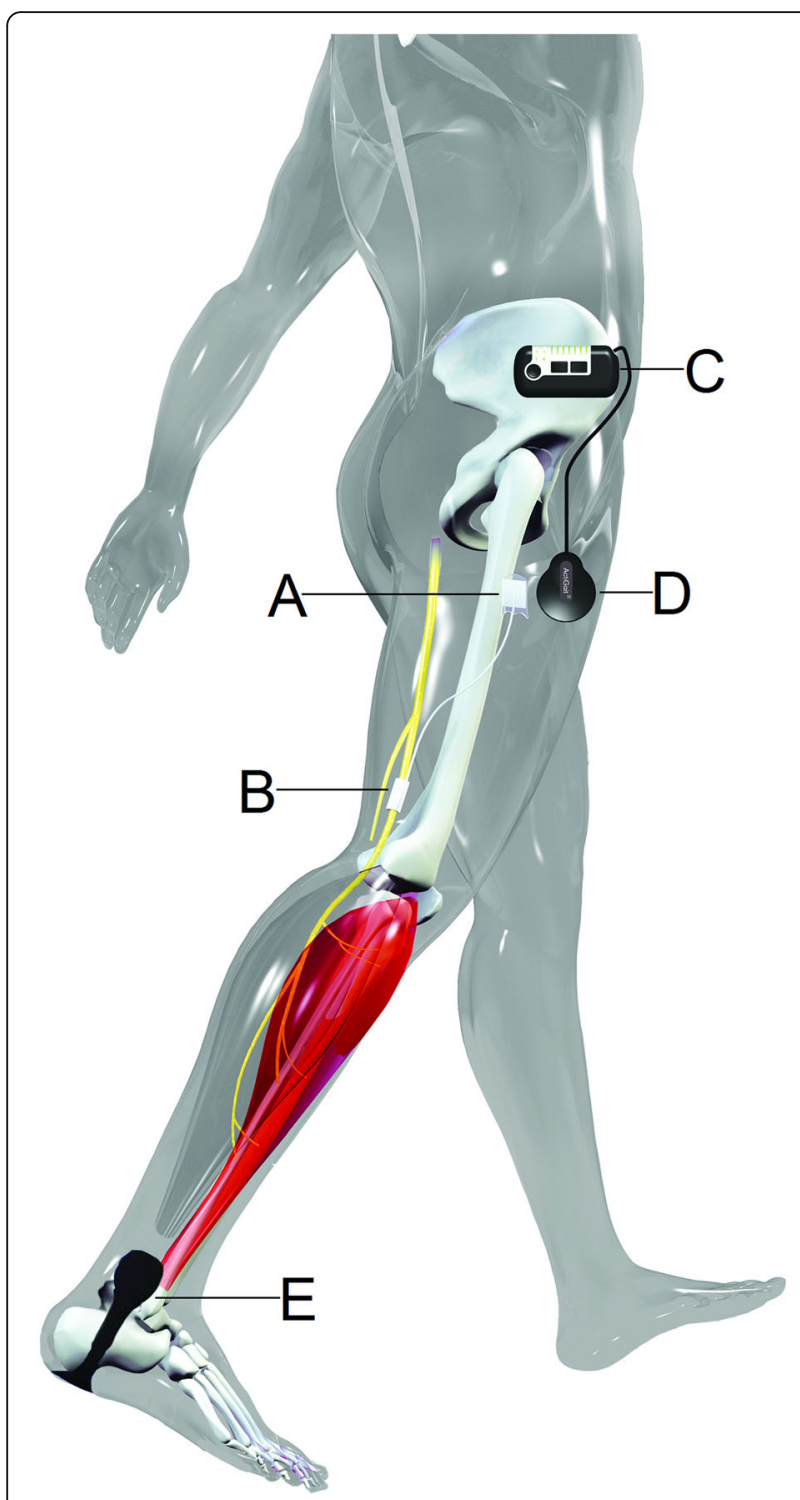

Fig. 2 The ActiGait ${ }^{\oplus}$ system. The implanted part of the ActiGait ${ }^{\oplus}$ consists of a stimulator body (a) and an electrode cuff (b), the latter placed around the common peroneal nerve. The implant is controlled by a control unit (c), which activates the implant through an antenna placed on the thigh (d). The timing of activation is determined by a heel switch (e), which communicates wirelessly with the control unit

an image histogram analysis tool, the mean gray value and thickness over the ROIs of separate muscles were calculated with the help of a custom software program ("QUMIA", [9, 19, 35-37]). This software then compared the muscle thickness and gray values to age-, sex-, heightand weight-corrected reference values [38,39]. The difference found was expressed as a z-score, i.e. the number of standard deviations (SD) from the mean. Positive z-scores for echogenicity (i.e. muscles looking relatively white on the screen) were considered indicative of poorer muscle architecture with more infiltration of fibrous tissue and fat. Negative z-scores for muscle thickness indicated loss of muscle mass.

\section{Electrophysiologic assessments}

Surface EMG signals were recorded from the tibialis anterior muscle on both the paretic and non-paretic side. Adhesive electrodes $(1 \mathrm{~cm}$ diameter $)$ were placed on the muscle belly and muscle tendon of the tibialis anterior muscle. Ground electrodes were placed between the stimulus and recording site. EMG recordings were performed using a multichannel biomedical amplifier (Neurotop MME 3132, Nihon Kohden, Tokyo, Japan).

\section{Peripheral motor nerve stimulation}

Peripheral motor nerve function was evaluated by measuring the CMAPs from the tibialis anterior muscle. Assessment of CMAPs from the tibialis anterior muscle has been shown to have good test-retest reliability [40]. CMAPs were obtained, for the paretic and non-paretic side separately, by stimulation of the common peroneal nerve, dorsal to the fibular head. Stimulation intensity was increased gradually until an increase in intensity did not further increase the motorwave (M-wave) amplitude (i.e. supramaximal stimulation). The results of five consecutive stimulations were then recorded and used for analysis. After rectification of the raw signal, the maximum peak-to-peak amplitude and the largest area under the curve (AUC) of these five contractions were extracted offline, using a custom Matlab script (Matlab 20141b, The Mathworks Inc. Natick, Massachusetts).

\section{Transcranial magnetic stimulation}

For assessment of the corticospinal connections to the tibialis anterior, MEPs were obtained using TMS. The motor cortex was stimulated three times at the vertex with a double-cone coil at maximal stimulator output using a transcranial magnetic stimulator (Magstim 200, Magstim, Whitland, UK). The MEPs were recorded when participants performed a slight voluntary ankle dorsiflexion to enlarge the MEP response. From the three stimuli obtained in each assessment, participants' maximum peak-to-peak amplitude as well as the largest AUC after rectification of the raw signal were extracted offline using a custom Matlab script (Matlab 2041b, The Mathworks Inc. Natick, Massachusetts). Since MEP output is limited by functioning of the lower motor neuron, MEP peak-to-peak amplitude and AUC were also normalized to CMAP amplitude and AUC, for instance: $\left(\mathrm{MEP}_{\text {amplitude }} / \mathrm{CMAP}_{\text {amplitude }}\right)^{*} 100 \%$.

\section{Statistical analysis}

To test whether group means of echogenicity and muscle thickness at baseline were different from normal 
values, a one sample t-test (test value: $\mu=0$ ) was performed. Baseline differences in echogenicity and muscle thickness between the paretic and non-paretic side were tested using paired samples t-tests. Generalized estimated equation modeling (GEE), with time (T0-T4) and side (paretic and non-paretic) as the independent variables, was used to assess changes over the follow-up period in the dependent variables: muscle echogenicity, muscle thickness, CMAP amplitude and AUC and (normalized) MEP amplitude and AUC. Since we expected that assessments obtained shortly after each other (e.g. T1 and T2) would be correlated stronger than assessments with a longer time interval (e.g. T2 and T3), we selected an autoregressive structure as the working correlation structure of the GEE model. In the case of a significant interaction of side by time, additional GEE analyses of time effects were performed for the paretic and non-paretic side, separately. These additional analyses of time per body side were corrected for multiple comparisons using Bonferroni correction. To assess changes in walking activity and capacity (steps per day and comfortable walking speed) Wilcoxon signed-rank tests were performed. We used SPSS (SPSS 15.0, SPSS Inc., Chicago, Illinois) for all statistical analyses. The level of significance was set at $p<0.05$.

\section{Results}

The characteristics of the included participants are presented in Table 1. One participant died before the first follow-up measurement, the cause of death being unrelated to the study; data of this participant was removed from the analysis. In one participant the FES system failed after 26 weeks. Since sufficient follow-up data were obtained, this participant was included in the final analysis. Hence, data of a total of 12 participants were used for final analysis. After 1 year of FES use the number of steps per day $(6248 \pm 3019, p=0.59)$ and comfortable

Table 1 Baseline group characteristics

\begin{tabular}{ll}
\hline $\mathrm{N}$ & 13 \\
\hline Age; mean yrs. (SD) & $56.1(10.2)$ \\
Sex; male/female & $10 / 3$ \\
Affected side; left/right & $8 / 5$ \\
Type of stroke; ischaemic/ haemorrhagic & $9 / 4$ \\
Years after stroke; mean yrs. (SD) & $5.2(4.5)$ \\
Body Mass Index; mean kg/m²(SD) & $26.7(3.4)$ \\
Fugl-Meyer Assessment - leg score & $71(53-85)$ \\
(0-100\%); median score (range) & \\
Motricity Index - leg score (0-100\%); & $72(42-91)$ \\
median score (range) & \\
Walking speed; mean m/s (SD) & $0.97(0.2)$ \\
Step count; mean steps/day (SD) & $5794(2671)$ \\
\hline
\end{tabular}

walking speed $(1.02 \pm 0.2 \mathrm{~m} / \mathrm{s}, p=0.09)$ were not significantly different from baseline values $(5794 \pm 2671$ and $0.97 \pm 0.2$, respectively).

\section{Quantitative muscle ultrasound Tibialis anterior}

At baseline, the mean echogenicity of the tibialis anterior muscle was significantly higher on both the paretic and non-paretic side $(\mathrm{z}=0.88, p=0.001$, and $\mathrm{z}=0.65, p=0.008$, respectively), meaning that the muscles looked more white and hence more structurally abnormal on the screen compared to reference values. Baseline echogenicity was not found to be significantly different between the paretic and non-paretic side. Over the follow-up period we found a decrease in tibialis anterior echogenicity which was most profound on the paretic side, as reflected by a significant interaction effect of time by side $(p<0.001)$. Echogenicity $\mathrm{z}$-scores on the paretic side were significantly different from baseline at T3 $(p<0.001)$ and T4 $(p<0.001)$ and decreased on average by $1.03 \mathrm{SD}$, indicating that muscle architecture improved over time. Remarkably, the echogenicity of the paretic side became lower than that of the nonparetic side at T4 (see Fig. 3a). We also found a significantly decreased echogenicity on the non-paretic side at $\mathrm{T} 4$ compared to $\mathrm{T} 0$, however, this finding was driven by imputation of two missing values at T4 by the GEE model. Additional parametric t-tests did not reveal a significant difference in echogenicity between baseline and T4 on the non-paretic side $(p=0.180)$.

At baseline, no significant differences compared to reference values or between the paretic and non-paretic side were found for muscle thickness of the tibialis anterior. Muscle thickness of the tibialis anterior on the paretic side increased over the follow-up period, being significantly different from baseline at T3 $(p<0.001)$ and T4 ( $p=0.001$; average increase of $0.80 \mathrm{SD}$, see Fig. $3 \mathrm{~b})$, whereas the muscle thickness on the non-paretic side did not (interaction time by side, $p<0.001$ ).

\section{Medial head of gastrocnemius}

At baseline, the mean echogenicity of the medial head of the gastrocnemius muscle was significantly higher on the paretic side compared to reference values $(p=0.019)$ and compared to the non-paretic side $(p=0.007)$. The mean echogenicity did not change over time for either the paretic or non-paretic side. Overall, mean z-scores for echogenicity of the paretic and non-paretic side were 1.59 and -0.13 , respectively (see Fig. 3c).

Similar to the echogenicity pattern of the gastrocnemius, the paretic side muscle thickness at baseline was significantly smaller than reference values $(p=0.002)$ and compared to the non-paretic side $(p=0.006)$. Over the follow-up period muscle thickness remained significantly different between the paretic and non-paretic side 


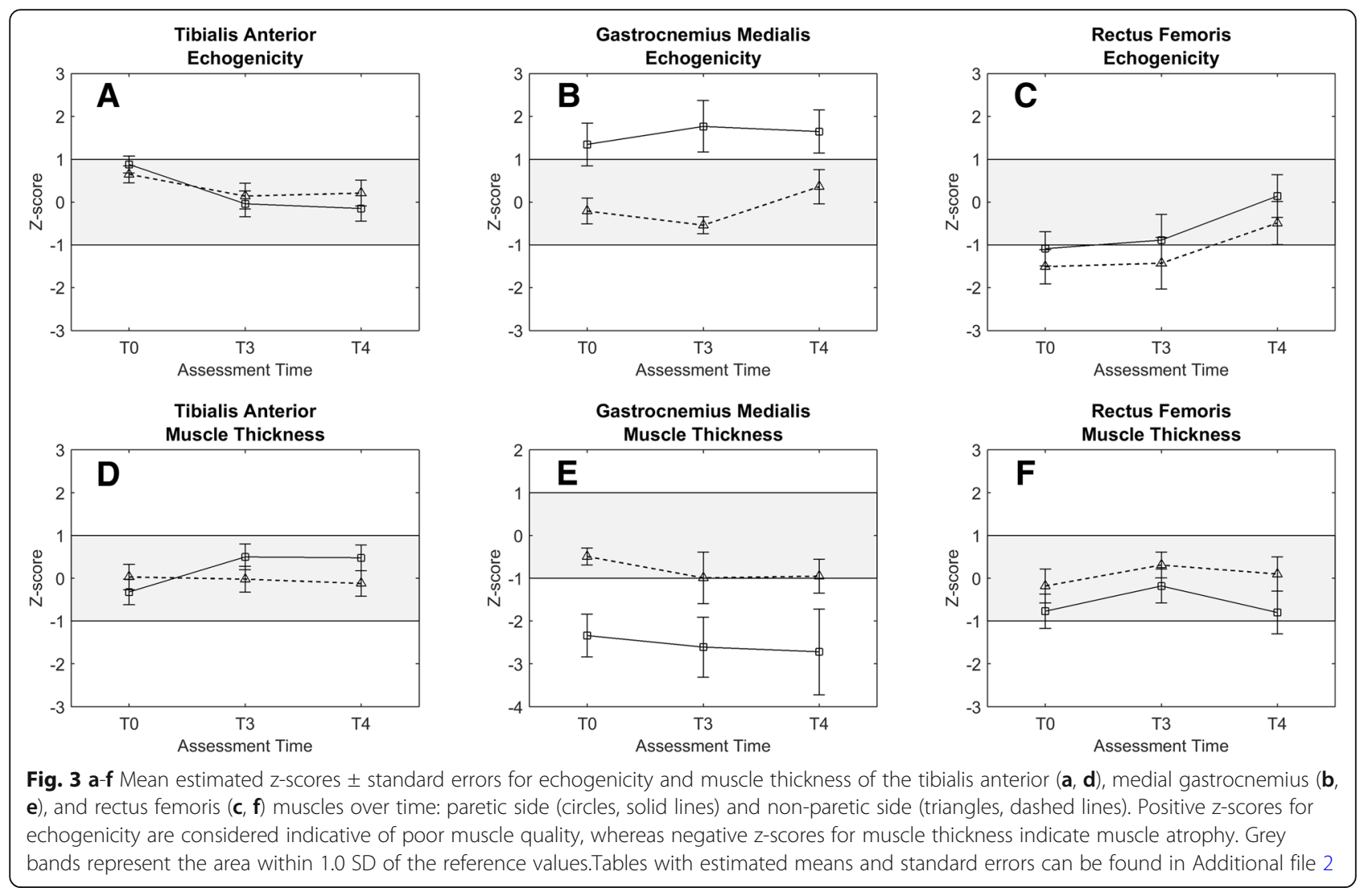

(mean muscle thickness $\mathrm{z}$-scores -2.56 and -0.81 , respectively, $p<0.001$ ), indicating that both sides showed reduced muscle thickness at a group level. Similar to the echogenicity, no significant changes in muscle thickness were found over time during FES use (see Fig. 3d).

\section{Rectus femoris}

At baseline, the mean echogenicity of the rectus femoris muscle was significantly lower than reference values on both the paretic side and non-paretic side $(\mathrm{z}=-1.09$, $p=0.014$, and $\mathrm{z}=-1.51, p=0.002$, respectively). Baseline echogenicity was not significantly different between the paretic and non-paretic side. During the follow-up period we found a significant change in echogenicity for both the paretic and non-paretic side at 52 weeks (T4) compared to T0 $(p=0.021)$. On average, echogenicity zscores increased by 1.24 and 1.01 for the paretic and non-paretic side, respectively. Thus, after 1 year of FES use, mean echogenicity values for both the paretic and non-paretic side returned within one SD of the reference values (see Fig. 3e).

At baseline, mean rectus femoris muscle thickness on the paretic and non-paretic side were not significantly different from reference values. Paretic side muscle thickness was significantly smaller compared to the nonparetic side $(p=0.009)$, which was maintained over the follow-up period $(p=0.001)$. On average, paretic and non-paretic z-scores were -0.58 and 0.08 , respectively, indicating that only the paretic side showed reduced muscle thickness. No significant changes in rectus femoris thickness on either side could be detected over time (see Fig. 3f).

\section{Electrophysiologic assessment Compound motor action potentials}

The CMAP AUC and amplitudes of the tibialis anterior muscle were generally lower on the paretic side compared to the non-paretic side $(p<0.001)$. Following FES implantation, differences in CMAP AUC between the paretic and non-paretic side became larger (interaction time by side $p<0.002$ ). This significant interaction of time by side was driven by a significant reduction in CMAP AUC on the paretic side (see Fig. 4a). It decreased from $58.7 \mathrm{mVms}$ (T0) to $46.0 \mathrm{mVms}$ (T4), and was found to be significantly different from baseline values during the entire follow-up period $(p<0.004)$. Similarly, an interaction of time by side was found for CMAP amplitudes $(p<0.005)$. Again, this interaction was driven by significant changes on the paretic side (see Fig. 4b). CMAP amplitudes on the paretic side decreased from $8.0 \mathrm{mV}$ (T0) to $6.4 \mathrm{mV}$ (T2, $p<0.001$ ). 

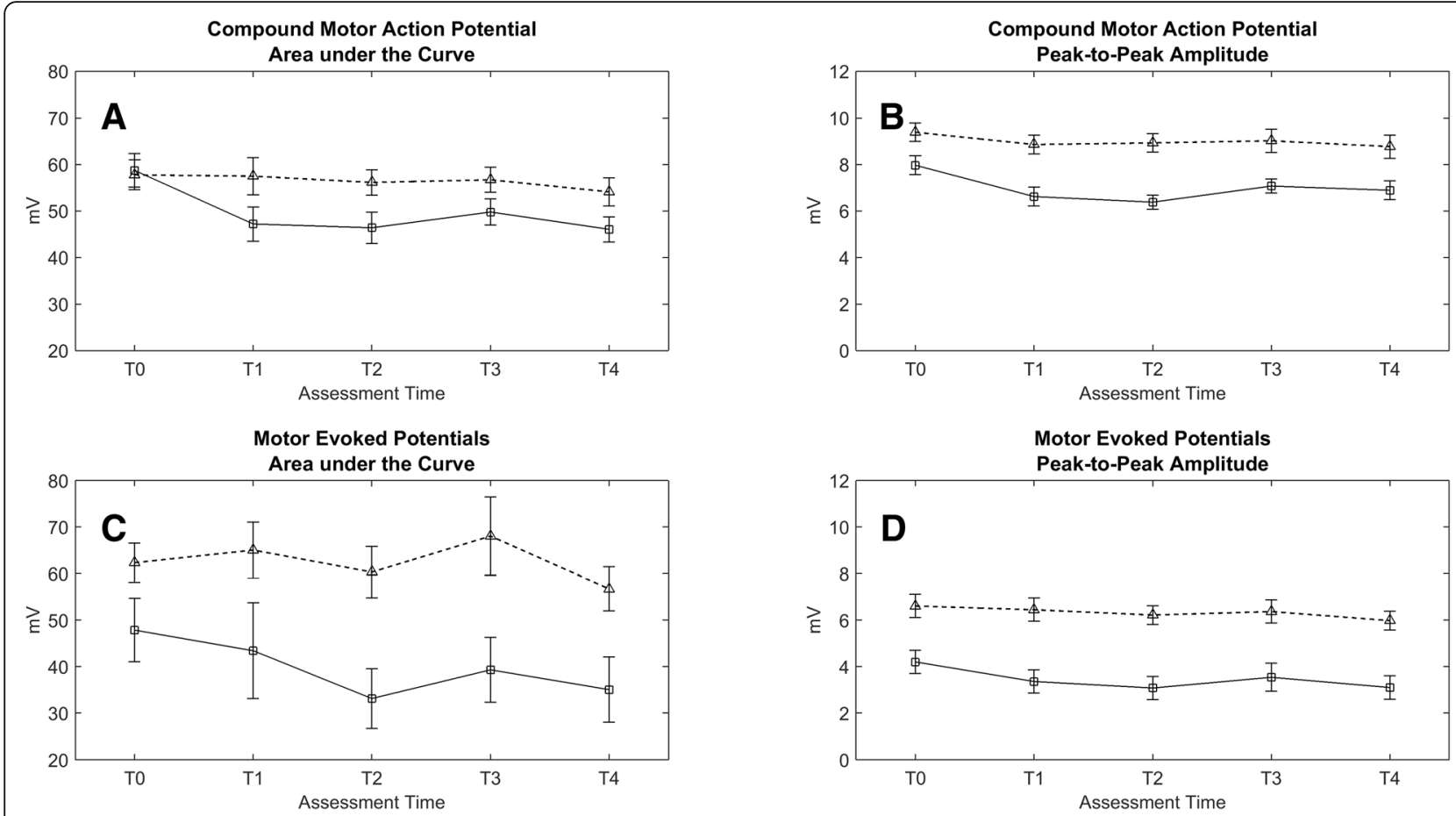

Fig. 4 a-d Mean estimated area under the curve and peak-to-peak amplitudes \pm standard errors for compound motor action potentials (a, b) and motor evoked potentials (c, $\mathbf{d}$ ) obtained from tibialis anterior muscle over time: paretic side (circles, solid lines) and non-paretic side (triangles, dashed lines). Tables with estimated means and standard errors can be found in Additional file 2

Subsequent analysis of the CMAP AUC and amplitudes showed no further changes from $\mathrm{T} 1$ or $\mathrm{T} 2$ to $\mathrm{T} 4$, indicating that the bilateral loss of CMAP amplitude/ AUC after the surgical intervention was not fully reversed during the 1-year follow-up period.

\section{Motor evoked potentials}

MEP AUC and amplitudes of the tibialis anterior muscle were generally lower on the paretic side compared to the non-paretic side $(p<0.036)$. Similar to the CMAP AUC and amplitudes, the differences in MEP AUC between the paretic and non-paretic side became larger during the follow-up period, as was reflected by a significant interaction of time by side $(p=0.005)$. Also for MEP AUC, this significant interaction of time by side was due to a decrease on the paretic side, which was significantly different from baseline at T2 $(p<0.001)$. Between baseline and T2, mean MEP AUC decreased from $47.9 \mathrm{mVms}$ to $33.1 \mathrm{mVms}$ on the paretic side (see Fig. 4c).

MEP amplitudes on the paretic and non-paretic side decreased from 4.2 to $3.1 \mathrm{mV}$ and from 6.6 to $6.0 \mathrm{mV}$, respectively. Although the average decrease in MEP amplitudes on the paretic side was nearly twofold the change on the non-paretic side,

no significant interaction of time by side was found ( $p=$ 0.119 at T2, see Fig. 4d). In general, MEP amplitudes were significantly different from baseline during the entire follow-up, T1-T4 $(p<0.046)$.

To assess whether changes over time in MEPs were due to changes in CMAPs we tested the effects of time and side on normalized MEPs. In contrast to MEP AUC, no significant interaction of time by side was found. Overall, normalized MEP AUC was significantly reduced, only at T2 $(p<0.001)$. With normalization of MEP amplitudes part of the time effects for MEP amplitudes, reported above, disappeared. With normalized MEP amplitudes significant differences in time were found only at T2 $(p<0.001)$ and T4 $(p=0.038)$.

\section{Discussion}

Our study confirms that structural changes in skeletal muscle after a supratentorial stroke, reported here and elsewhere [9, 41-45], appear to be reversible with longterm FES use. Echogenicity of the tibialis anterior muscle decreased during the follow-up on the paretic side, which was accompanied by an increase in muscle size. Because of the chronic condition of the participants, it is unlikely that these changes are attributable to spontaneous recovery.

\section{Quantitative muscle ultrasound}

In agreement with our hypothesis, the year-long use of an implanted peroneal FES stimulator successfully reversed the maladaptive changes to muscle structure in the tibialis 
anterior muscle. Previous studies have shown that FES can be successful in stopping or counteracting muscle atrophy, but this was done in other groups of patients (e.g., spinal cord injury) and through a resistance training program specifically aimed at muscle mass gain using surface-based FES stimulation [46-48]. The reduction in echogenicity on the paretic side of 1.03 SD comes down to a change in absolute echogenicity of approximately $20 \%$ [38], which is well over the $10 \%$ variation that could be expected between measurements [31]. Reduced echogenicity in the tibialis anterior muscle may have (partly) been caused by an increase in muscle mass [49], something we observed on the paretic side. However, the changes in echogenicity on the paretic side exceeded the changes in muscle thickness, which makes it unlikely that the observed decrease in echogenicity would be merely due to activity-induced muscle hypertrophy.

Since the implanted FES stimulator acts on the ankle dorsiflexor muscles, we hypothesized that no significant changes would occur in the medial gastrocnemius or rectus femoris muscle during 1 year of FES use. Although this was true for the medial head of the gastrocnemius, we did find changes in rectus femoris echogenicity during the follow-up period. Unexpectedly, and unlike the other muscles, the average echogenicity of the rectus femoris muscle was bilaterally lower than the reference values at baseline. This finding contrasts with the recent work by Akazawa et al. who reported increased echogenicity in the paretic rectus femoris muscles of sedentary and active people with stroke [45]. However, in the study by Akazawa et al., echogenicity was not corrected for age, sex, height or weight, as we did in the current study. As a result, direct comparison of the two studies is not possible. We have no explanation for the relatively low echogenicity of both rectus femoris muscles as observed in our study, however, our results indicate that this echogenicity was normalized after one-year of FES use. The normalization of echogenicity might point at a changed activity of the rectus femoris muscles as a result of FES use, at least on the paretic side, but this post-hoc explanation needs further investigation.

\section{Neurophysiologic assessment}

Our results indicate that the CMAPs from the tibialis anterior muscle of the stimulated leg decreased after implantation of the FES system, a finding which we did not expect. The marked decline of the CMAP amplitudes on the paretic side (i.e. a loss of about $20 \%$ ) could theoretically result from a reduction of functionally active motor units (e.g. due to nerve damage upon implantation) or from a lower excitability of motor unit components (i.e. axons, neuromuscular junctions and/or muscle fibers). Given the invasive character of the intervention, it is possible that the common peroneal nerve might have been damaged during surgery. However, if this would have been the case, we would have expected problems stimulating the tibialis anterior muscles and we would have expected signs of denervation of this muscle (i.e., increase in echogenicity and decrease in muscle size), which we did not find. We therefore believe that it is most likely that the excitability of the common peroneal nerve changed following implantation and, particularly, following activation of the FES stimulator. Indeed, axonal excitability can change under various conditions including overuse and fatigue [50, 51], and rapidly fatiguing muscles are a well-known problem in the application of FES [52]. Although participants were instructed to build up their daily FES use slowly and gradually, it might be that the paretic neuromuscular complex needed more time or was unable to adjust to the suddenly increased activation. Signs of fatigue were commonly reported by the participants, especially during the first weeks of follow-up. Additionally, it might be that the nature of FES provides a very different stimulation context for the motor axons than physiologic activation does, with concomitant changes in axonal resting and firing thresholds that may lead to a decrease in single-stimulus CMAP parameters.

In a number of studies, the use of FES has been shown to induce plastic changes on a cortical level [13-16]. Like in our study, Everaert et al. used MEPs to assess the effects of FES on cortical plasticity. In contrast to their work, we did not find signs of cortical plasticity after a year-long FES use. Instead, in our study, MEP amplitudes from the tibialis anterior muscle were even slightly reduced during the follow-up period. However, direct comparison of the results derived from both studies should be done with caution, given an important difference in measurement protocol. Where we used a fixed position of the magnetic stimulator for eliciting MEPs throughout the follow-up period, Everaert et al. aimed for the localization of 'hot spots' to achieve the best MEP response.

\section{Study limitations and recommendations}

We assessed changes in three muscles in a relatively small and specific group of chronic stroke patients. The participants in our sample showed reduced ambulatory capacity, but were relatively active compared to the stroke community at large. The group in our study walked on average about 5800 steps per day, which is well above the number of steps reported in other studies [53]. To assure reliable imaging of muscles with muscle ultrasound, stroke survivors who were morbidly obese were excluded from the study. Since people with stroke are often obese and inactive, our results may therefore not be representative for the entire group of stroke survivors. However, our data show that even in active people with stroke structural changes in muscle architecture can be reversed, in our case by applying peroneal 
FES. Although we compared muscle characteristics to height-, weight-, sex- and age-corrected reference values, it is still uncertain whether the changes obtained in our study are clinically relevant. Future studies should aim at identifying cut-off criteria for relevant changes in both echogenicity and muscle thickness in this study population. In addition, other factors might influence muscle ultrasound characteristics in people with stroke, such as their hydration status that can change with the use of diuretics. The effects of such variables should be investigated in future research. Finally, this study focused on reversing the structural changes to muscle and nerves in people with chronic stroke ( $>6$ months post onset). It might be interesting to investigate whether FES could also be used to prevent such structural changes in the first months after a stroke.

\section{Conclusion}

We have shown that the structural changes to muscles following supratentorial stroke are reversible with implanted peroneal FES and that these findings are not restricted to the stimulated ankle dorsiflexor muscles alone. We could not identify improvement of lower motor neuron functioning or cortical plasticity. The findings in this study add to the evidence that peroneal FES may have added value over the use of an ankle-foot orthosis in people with unilateral drop foot after stroke.

\section{Additional files}

Additional file 1: Detailed description of the surgical procedure and system activation. (DOCX $17 \mathrm{~kb}$ )

Additional file 2: Tables with estimated means and standard errors on which the figures in the main manuscript were based. (DOCX 20 kb)

\section{Abbreviations}

AUC: Area under the curve; CMAPs: Compound motor action potentials; EMG: Electromyography; FES: Functional electrical stimulation; GEE: Generalized estimated equations; LMN: Lower motor neuron; MEPs: Motor evoked potentials; M-wave: Motor-wave; QMUS: Quantitative muscle ultrasound; ROI: Region of interest; UMN: Upper motor neuron

\section{Acknowledgements}

We thank J. de Vries, and T. Beemst for their neurosurgical contribution and J. den Boer, R. van Swigchem, G van Bon and W. Raijmann for their help during the measurements.

\section{Authors' contributions}

SG, NVA and WW designed the study. SG included the participants and assessed clinimetrics at baseline and 52 weeks after implantation. FB performed neurophysiologic measurements and collected QMUS data. FB, W, SG and NVA interpreted the data and drafted the manuscript. All authors read and approved the final manuscript.

\section{Funding}

The study was financed by the TWIN Institute for Neuromodulation in the Netherlands. The funding body was not involved in the design of the study, data collection, data analysis, data interpretation and preparation of the manuscript.

\section{Availability of data and materials}

The datasets used and/or analysed during the current study are available from the corresponding author on reasonable request.

\section{Ethics approval and consent to participate}

All patients gave their informed consent. The study was approved by the local medical ethics committee (CMO regio Arnhem - Nijmegen) and conducted in accordance with the World Medical Association Declaration of Helsinki [23].

\section{Consent for publication}

Not applicable.

\section{Competing interests}

The authors declare that they have no competing interests.

\section{Author details}

'Department of Rehabilitation, Donders Institute for Brain, Cognition and Behaviour, Radboud University Medical Center, P.O. Box 9101, 6500, HB, Nijmegen, The Netherlands. ${ }^{2}$ Department of Neurology and Clinical Neurophysiology, Donders Institute for Brain, Cognition and Behaviour, Radboud University Medical Center, P.O. Box 9101, 6500, HB, Nijmegen, The Netherlands.

Received: 20 March 2019 Accepted: 21 June 2019

Published online: 10 July 2019

\section{References}

1. Benecke R, Berthold A, Conrad B. Denervation activity in the EMG of patients with upper motor neuron lesions: time course, local distribution and pathogenetic aspects. J Neurol. 1983;230(3):143-51.

2. Spaans F, Wilts G. Denervation due to lesions of the central nervous system. An EMG study in cases of cerebral contusion and cerebrovascular accidents. J Neurol Sci. 1982;57(2-3):291-305.

3. van Kuijk AA, Pasman JW, Hendricks HT, Zwarts MJ, Geurts AC. Predicting hand motor recovery in severe stroke: the role of motor evoked potentials in relation to early clinical assessment. Neurorehabil Neural Repair. 2009; 23(1):45-51.

4. Hara Y, Masakado Y, Chino N. The physiological functional loss of single thenar motor units in the stroke patients: when does it occur? Does it progress? Clin Neurophysiol. 2004;115(1):97-103.

5. Lukacs M. Electrophysiological signs of changes in motor units after ischaemic stroke. Clin Neurophysiol. 2005;116(7):1566-70.

6. Scherbakov N, Sandek A, Doehner W. Stroke-related sarcopenia: specific characteristics. J Am Med Dir Assoc. 2015;16(4):272-6.

7. English C, McLennan $\mathrm{H}$, Thoirs $\mathrm{K}$, Coates A, Bernhardt J. Loss of skeletal muscle mass after stroke: a systematic review. Int J Stroke. 2010;5(5): 395-402.

8. Hafer-Macko CE, Ryan AS, Ivey FM, Macko RF. Skeletal muscle changes after hemiparetic stroke and potential beneficial effects of exercise intervention strategies. J Rehabil Res Dev. 2008;45(2):261-72.

9. Berenpas F, Martens AM, Weerdesteyn V, Geurts AC, van Alfen N. Bilateral changes in muscle architecture of physically active people with chronic stroke: a quantitative muscle ultrasound study. Clin Neurophysiol. 2017; 128(1):115-22

10. Dunning K, O'Dell MW, Kluding P, McBride K. Peroneal stimulation for foot drop after stroke: a systematic review. Am J Phys Med Rehabil. 2015;94(8): 649-64.

11. Liberson WT, Holmquest HJ, Scot D, Dow M. Functional electrotherapy: stimulation of the peroneal nerve synchronized with the swing phase of the gait of hemiplegic patients. Arch Phys Med Rehabil. 1961;42:101-5.

12. Khaslavskaia S, Sinkjaer T. Motor cortex excitability following repetitive electrical stimulation of the common peroneal nerve depends on the voluntary drive. Exp Brain Res. 2005;162(4):497-502.

13. Everaert DG, Thompson AK, Chong SL, Stein RB. Does functional electrical stimulation for foot drop strengthen corticospinal connections? Neurorehabil Neural Repair. 2010;24(2):168-77.

14. Thibaut A, Moissenet F, Di Perri C, Schreiber C, Remacle A, Kolanowski E, et al. Brain plasticity after implanted peroneal nerve electrical stimulation to improve gait in chronic stroke patients: two case reports. NeuroRehabilitation. 2017:40(2):251-8. 
15. Merkel C, Hausmann J, Hopf JM, Heinze HJ, Buentjen L, Schoenfeld MA. Active prosthesis dependent functional cortical reorganization following stroke. Sci Rep. 2017;7(1):8680.

16. Stein RB, Chong S, Everaert DG, Rolf R, Thompson AK, Whittaker M, et al. A multicenter trial of a footdrop stimulator controlled by a tilt sensor. Neurorehabil Neural Repair. 2006;20(3):371-9.

17. Pillen S, Tak RO, Zwarts MJ, Lammens MM, Verrijp KN, Arts IM, et al. Skeletal muscle ultrasound: correlation between fibrous tissue and echo intensity. Ultrasound Med Biol. 2009;35(3):443-6.

18. Mul K, Horlings CGC, Vincenten SCC, Voermans NC, van Engelen BGM, van Alfen N. Quantitative muscle MRI and ultrasound for facioscapulohumeral muscular dystrophy: complementary imaging biomarkers. J Neurol. 2018; 265(11):2646-55.

19. Arts IMP, Overeem S, Pillen S, Jurgen Schelhaas H, Zwarts MJ. Muscle changes in amyotrophic lateral sclerosis: a longitudinal ultrasonography study. Clin Neurophysiol. 2011;122(3):623-8.

20. Pillen $\mathrm{S}$, Arts IM, Zwarts MJ. Muscle ultrasound in neuromuscular disorders. Muscle Nerve. 2008;37(6):679-93.

21. Arts IM, van Rooij FG, Overeem S, Pillen S, Janssen HM, Schelhaas HJ, et al. Quantitative muscle ultrasonography in amyotrophic lateral sclerosis. Ultrasound Med Biol. 2008;34(3):354-61.

22. Berenpas F, Schiemanck S, Beelen A, Nollet F, Weerdesteyn V, Geurts A. Kinematic and kinetic benefits of implantable peroneal nerve stimulation in people with post-stroke drop foot using an ankle-foot orthosis. Restor Neurol Neurosci. 2018;36(4):547-58.

23. World Medical A. World Medical Association Declaration of Helsinki. Ethical principles for medical research involving human subjects. Bull World Health Organ. 2001;79(4):373-4.

24. Demeurisse G, Demol O, Robaye E. Motor evaluation in vascular hemiplegia. Eur Neurol. 1980;19(6):382-9.

25. Fugl-Meyer AR, Jaasko L, Leyman I, Olsson S, Steglind S. The post-stroke hemiplegic patient. 1. A method for evaluation of physical performance. Scand J Rehabil Med. 1975;7(1):13-31

26. Peters DM, Fritz SL, Krotish DE. Assessing the reliability and validity of a shorter walk test compared with the 10-meter walk test for measurements of gait speed in healthy, older adults. J Geriatr Phys Ther. 2013;36(1):24-30.

27. Burridge $\mathrm{JH}$, Haugland M, Larsen B, Pickering RM, Svaneborg N, Iversen HK, et al. Phase II trial to evaluate the ActiGait implanted drop-foot stimulator in established hemiplegia. J Rehabil Med. 2007;39(3):212-8.

28. Ernst J, Grundey J, Hewitt M, von Lewinski F, Kaus J, Schmalz T, et al. Towards physiological ankle movements with the ActiGait implantable drop foot stimulator in chronic stroke. Restor Neurol Neurosci. 2013;31(5):557-69.

29. Schiemanck $S$, Berenpas F, van Swigchem $R$, van den Munckhof $P$, de Vries J, Beelen A, et al. Effects of implantable peroneal nerve stimulation on gait quality, energy expenditure, participation and user satisfaction in patients with post-stroke drop foot using an ankle-foot orthosis. Restor Neurol Neurosci. 2015:33(6):795-807.

30. Pillen S, van Keimpema M, Nievelstein RA, Verrips A, van KruijsbergenRaijmann W, Zwarts MJ. Skeletal muscle ultrasonography: Visual versus quantitative evaluation. Ultrasound Med Biol. 2006;32(9):1315-21.

31. O'Brien TG, Cazares Gonzalez ML, Ghosh PS, Mandrekar J, Boon A Reliability of a novel ultrasound system for gray-scale analysis of muscle. Muscle Nerve. 2017:56(3):408-12.

32. Zaidman CM, Wu JS, Wilder S, Darras BT, Rutkove SB. Minimal training is required to reliably perform quantitative ultrasound of muscle. Muscle Nerve. 2014;50(1):124-8.

33. van Alfen N, Mah JK. Neuromuscular ultrasound: a new tool in your toolbox. Can J Neurol Sci. 2018;45(5):504-15.

34. Scholten RR, Pillen S, Verrips A, Zwarts MJ. Quantitative ultrasonography of skeletal muscles in children: normal values. Muscle Nerve. 2003;27(6):693-8

35. Jansen M, van Alfen N, Nijhuis van der Sanden MW, van Dijk JP, Pillen S, de Groot IJ. Quantitative muscle ultrasound is a promising longitudinal follow-up tool in Duchenne muscular dystrophy. Neuromuscul Disord. 2012;22(4):306-17.

36. Janssen $B H$, Pillen $S$, Voet NB, Heerschap A, van Engelen BG, van Alfen N Quantitative muscle ultrasound versus quantitative magnetic resonance imaging in facioscapulohumeral dystrophy. Muscle Nerve. 2014;50(6):968-75.

37. Bickerstaffe A, Beelen A, Zwarts MJ, Nollet F, van Dijk JP. Quantitative muscle ultrasound and quadriceps strength in patients with post-polio syndrome. Muscle Nerve. 2015;51(1):24-9.

38. Arts IM, Pillen S, Schelhaas HJ, Overeem S, Zwarts MJ Normal values for quantitative muscle ultrasonography in adults. Muscle Nerve. 2010;41(1):32-41.
39. Verhulst FV, Leeuwesteijn AE, Louwerens JW, Geurts AC, Van Alfen N, Pillen S. Quantitative ultrasound of lower leg and foot muscles: feasibility and reference values. Foot Ankle Surg. 2011;17(3):145-9.

40. Chaudhry V, Cornblath DR, Mellits ED, Avila O, Freimer ML, Glass JD, et al. Inter- and intra-examiner reliability of nerve conduction measurements in normal subjects. Ann Neurol. 1991;30(6):841-3.

41. Picelli A, Tamburin S, Cavazza S, Scampoli C, Manca M, Cosma M, et al. Relationship between ultrasonographic, electromyographic, and clinical parameters in adult stroke patients with spastic equinus: an observational study. Arch Phys Med Rehabil. 2014;95(8):1564-70.

42. Ryan AS, Buscemi A, Forrester L, Hafer-Macko CE, Ivey FM. Atrophy and intramuscular fat in specific muscles of the thigh: associated weakness and hyperinsulinemia in stroke survivors. Neurorehabil Neural Repair. 2011;25(9): 865-72.

43. Ryan AS, Dobrovolny $\mathrm{CL}$, Smith GV, Silver KH, Macko RF. Hemiparetic muscle atrophy and increased intramuscular fat in stroke patients. Arch Phys Med Rehabil. 2002;83(12):1703-7.

44. Klein CS, Brooks D, Richardson D, Mcllroy WE, Bayley MT. Voluntary activation failure contributes more to plantar flexor weakness than antagonist coactivation and muscle atrophy in chronic stroke survivors. J Appl Physiol (1985). 2010;109(5):1337-46.

45. Akazawa N, Harada K, Okawa N, Tamura K, Hayase A, Moriyama H. Relationships between muscle mass, intramuscular adipose and fibrous tissues of the quadriceps, and gait independence in chronic stroke survivors: a cross-sectional study. Physiotherapy. 2018;104(4):438-45.

46. Dudley-Javoroski S, McMullen T, Borgwardt MR, Peranich LM, Shields RK. Reliability and responsiveness of musculoskeletal ultrasound in subjects with and without spinal cord injury. Ultrasound Med Biol. 2010;36(10):1594-607.

47. Ryan TE, Brizendine JT, Backus D, McCully KK. Electrically induced resistance training in individuals with motor complete spinal cord injury. Arch Phys Med Rehabil. 2013;94(11):2166-73.

48. Kern H, Carraro U, Adami N, Biral D, Hofer C, Forstner C, et al. Home-based functional electrical stimulation rescues permanently denervated muscles in paraplegic patients with complete lower motor neuron lesion. Neurorehabil Neural Repair. 2010;24(8):709-21.

49. Jacobs J, Jansen M, Janssen H, Raijmann W, Van Alfen N, Pillen S. Quantitative muscle ultrasound and muscle force in healthy children: a 4year follow-up study. Muscle Nerve. 2013;47(6):856-63.

50. Burke D, Kiernan MC, Bostock H. Excitability of human axons. Clin Neurophysiol. 2001;112(9):1575-85.

51. Estigoni EH, Fornusek C, Hamzaid NA, Hasnan N, Smith RM, Davis GM. Evoked EMG versus muscle torque during fatiguing functional electrical stimulation-evoked muscle contractions and short-term recovery in individuals with spinal cord injury. Sensors (Basel). 2014;14(12):22907-20.

52. Ibitoye MO, Hamzaid NA, Hasnan N, Abdul Wahab AK, Davis GM. Strategies for rapid muscle fatigue reduction during FES exercise in individuals with spinal cord injury: a systematic review. PLoS One. 2016;11(2):e0149024.

53. Michael KM, Allen JK, Macko RF. Reduced ambulatory activity after stroke: the role of balance, gait, and cardiovascular fitness. Arch Phys Med Rehabil. 2005;86(8):1552-6.

\section{Publisher's Note}

Springer Nature remains neutral with regard to jurisdictional claims in published maps and institutional affiliations.

Ready to submit your research? Choose BMC and benefit from:

- fast, convenient online submission

- thorough peer review by experienced researchers in your field

- rapid publication on acceptance

- support for research data, including large and complex data types

- gold Open Access which fosters wider collaboration and increased citations

- maximum visibility for your research: over $100 \mathrm{M}$ website views per year

At $\mathrm{BMC}$, research is always in progress.

Learn more biomedcentral.com/submission 